\title{
Diversity and population structure of black soybean landraces originating from Tanba and neighboring regions
}

\author{
Tomoko Hirota1), Takashi Sayama ${ }^{2)}$, Masanori Yamasaki'3), Hiroko Sasama ${ }^{2)}$, Takuma Sugimoto4), \\ Masao Ishimoto ${ }^{2)}$ and Shinya Yoshida*4,5) \\ 1) Hokubu Agricultural Institute, Hyogo Prefectural Research Center of Agriculture, Forestry and Fisheries, Asago, Hyogo 669-5254, \\ Japan \\ 2) National Institute of Agrobiological Sciences, 2-1-2 Kannondai, Tsukuba, Ibaraki 305-8602, Japan \\ 3) Food Resources Education and Research Center, Kobe University, Kasai, Hyogo 675-2103, Japan \\ 4) Hyogo Prefectural Research Center of Agriculture, Forestry and Fisheries, Kasai, Hyogo 679-0198, Japan \\ 5) Graduate school of agriculture, Kobe University, Rokkoudai, Nada, Kobe, Hyogo 675-8501, Japan
}

\begin{abstract}
Black soybean landraces that had been cultivated in Tanba region and the neighboring regions and conserved black soybean landraces, including those from other regions in Japan, were used in this study. The polymorphisms of 78 SSR markers in nuclear DNA and 6 SSRs in chloroplast DNA were analyzed in the black soybean landrace populations. The result of phylogenic analysis revealed that the black soybeans can be classified into six clades. The landraces originating from Tanba region were classed into first and second clades, and two chloroplast genotypes were found in the population of black soybeans from the Tanba region. Genotype A chloroplast was predominantly identified in major populations of the Tanba, while genotype B was widely distributed in the black soybean population. Population structure analysis in the Japanese black soybean accessions inferred there are six groups. The black soybean landrace from the Tanba region was classified into three groups, mainly corresponding to the distance-based phylogenic results. The two groups were probably derived from different ancestors with Type A and B chloroplast genomes, respectively, whereas the other group showed both types of chloroplast genome. The admixture situations suggested that the landraces in the main group have been widely cultivated in Tanba region, while the landraces that belong to other groups were cultivated in localized area. Several phenotypes were compared among genotype groups, dividing into two sub-groups: founder sub-group and admixed sub-group. Phenotypic differences were observed between founder landraces in group 1 and group 3. On the other hand, landraces in admixture landraces in group 1 and group 2 segregated for several traits, while founder landraces in group 1 were stabled for each trait. These observations suggest that gene flow events have occurred between different founder landraces.
\end{abstract}

Key Words: black soybean landrace, SSR, genetic diversity, Glycine max (L.).

\section{Introduction}

Black soybeans cultivated in the Tanba region (including Tanba and neighboring regions) in Hyogo and Kyoto prefectures, Japan, are characterized by extremely large seeds suitable for boiled bean processing as agricultural products and autumn soybean with extremely late filling. The weight of 100 seeds of "Tanbaguro" usually achieves more than $80 \mathrm{~g}$, larger than all other soybean varieties. Previously, black soybean landraces have been called by various names in each place or community where they are cultivated in Tanba region, but "Tanbaguro" is now used as the collective name for landraces originating from Tanba region, although the

Communicated by Duncan Vaughan

Received July 20, 2011. Accepted October 14, 2011.

*Corresponding author (e-mail: Shinya_Yoshida01@pref.hyogo.lg.jp) genetic background of the landrace population is unclear. Nagata (1953) collected landraces cultivated in the main production area in Tanba region and surveyed the seed characteristics. As a result, he reported that the weight of 100 seeds ranged from 45 to $65 \mathrm{~g}$, which was attributed not only to the cultivation conditions but also to the genetic background. Until recently, in Tanba region, black soybean landraces were selected and maintained by farmers using saved seeds; therefore, it is considered that these landraces differ among farmers/communities growing black soybeans. Later, by pure-line isolation, "Shin-Tanbaguro" and "HyoukeiKuro 3" were selected and spread to become dominant varieties in Kyoto and Hyogo prefectures, respectively.

In other parts of Japan various black soybean varieties are cultivated and vary in growth characteristics and/or morphology. For example, "Chuusei-Hikariguro" is commonly cultivated in Hokkaido, has smaller seeds than "Tanbaguro" and 
is not covered with bloom on the seed coat like "Tanbaguro". Furthermore, several cultivars in eastern Japan are characterized by having flat seeds; however, the genetic relationship among these black soybean varieties and the classification of landraces in Tanba region has been little studied.

The genetic diversity of soybean in Far East Asia, North America and Japan were investigated using the isozyme pattern and several molecular markers (George et al. 2003, Hirata et al. 1999, Hwang et al. 2008, Wang et al. 2006). At a result, it is considered that the Japanese soybeans are different from the Chinese soybeans. For classification of 87 Japanese soybean cultivars and wild soybeans, Hwang et al. (2008) analyzed the length polymorphism of 377 SSR markers and classified them into three groups. It was reported that "Tanbaguro" was included in sub-cluster 1a in this study, and this sub-cluster included Japanese cultivars from around the country with the exception of Hokkaido (Hwang et al. 2008). On the other hand, Hatanaka et al. (2008) analyzed 20 accessions, including "Tanbaguro" and other types of black soybean varieties and yellow soybean varieties using 23 SSR markers. As a result, "Tanbaguro" landraces were distinguished from other black soybean varieties from Hokkaido and Eastern Japan. Kosaka et al. (2009) reported how to identify "Tanbaguro" as an ingredient of processed foods by SSR analysis. These reports did not clarify the genetic diversity and differentiation of the black soybeans originating from Tanba and neighboring regions, although the major landrace of "Tanbaguro" could be discriminated from other black soybean varieties and imported products.

We collected black soybean landraces that had been cultivated in Tanba and neighboring regions and conserved at several organizations, and investigated their SSR polymorphism in this study. Using the SSR polymorphism, the classification and phylogenic relationships have been calculated using polymorphism similarity among varieties. Recently, population structure analysis was performed for several species by DNA polymorphism. This method is useful for evolutionary investigation of genetic exchange between populations. We investigated whether the diversity of black soybean populations in Tanba region is derived from different populations. Furthermore, the sub-populations classified by genetic diversity were characterized by their growth characteristics and seed morphology.

\section{Materials and Methods}

\section{Plant materials}

Landraces were collected from fields in Tanba and neighboring regions and also obtained from conserved and/or distributed lines from Hyogo and Kyoto prefectural experimental stations, National Institute of Agrobiological Sciences Genebank, Kyoto University and Odagaki, Inc. Classification of accessions by origin is shown (Fig. 1). These landraces were cultivated at Hyogo Prefectural Research Center for Agriculture (Kasai, Hyogo, Japan) and seeds were obtained from individuals in 2008. Selected lines by characteristic

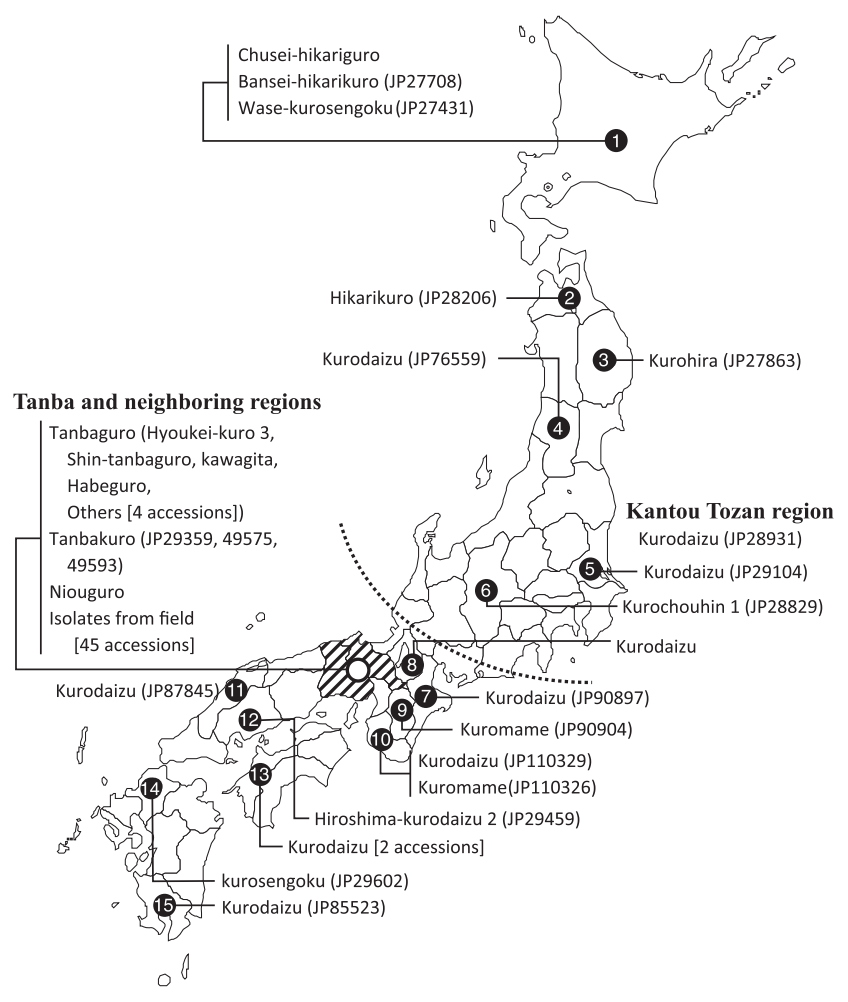

Fig. 1. Geographical distribution of 76 black soybean landraces in this study. JP no. in round bracket indicate accessions number at National Institute of Agrobiological Sciences. The number with solid circle specifies the prefecture of each landrace origin. 1: Hokkaido, 2: Aomori, 3: Iwate, 4: Yamagata, 5: Ibaragi, 6: Nagano, 7: Mie, 8: Shiga, 9: Nara, 10: Wakayama, 11: Shimane, 12: Hiroshima, 13: Ehime, 14: Fukuoka, 15: Kagoshima. Eastern Japan specifies the range form 1 to 6 of prefectures, and western Japan specifies the range form 7 to 15 of prefectures. Tanba and neighboring regions are specified a part of Hyogo and Kyoto prefecture that shaded by oblique line. Accession of JP28931 is originated from eastern Japan (Kanto Tozan region), but prefecture of origin is unknown. A broken line indicate the boundary line between eastern and western Japan.

and SSR marker genotypes were cultivated to investigate the growth characteristics and seed shape at Kasai in 2009. For phenotypic measurement, the seeds were sown in 76 cell trays with nursery soil on 12 June, and nursery plants were transplanting in a plot with rows of $120 \mathrm{~cm}$ length spaced $45 \mathrm{~cm}$ apart in the field.

\section{Phenotypic measurements}

For the growth characteristics, the flowering period, maturity period, stem length, branch number, node number, pod number and seed weight were measured in five plants in each line. The flowering period was counted as the number of days from sowing to the time at $50 \%$ flowering. Each plant was measured for its branch number (branches with more than two nodes), stem length (distance from the cotyledonary node to the tip of the main stem), node number for the main stem and pod number with seeds.

The dimensions of harvested seeds were measured as 
Table 1. SSR marker panel for multiplex PCR

\begin{tabular}{ll}
\hline \hline Marker panel name & \\
\hline Chr01 & SSR marker \\
Chr02 & Satt184, Sat_353, Satt370, Satt129 \\
Chr03 & Satt216, Sat_211, Satt141, Satt459 \\
Chr04 & Sct_195, Satt530, Satt237, Sat_125 \\
Chr05 & Satt565, Sat_337, Satt646, AI794821 \\
Chr06 & Sat_137, Sat_344, Satt545, Satt211 \\
Chr07 & Satt281, Satt322, Satt277, Satt307 \\
Chr08 & Satt150, Satt536, Satt346, Sat_330 \\
Chr09 & Sat_409, Sat_115, Satt329, Satt378 \\
Chr10 & Satt349, Satt499, Satt196, Satt588 \\
Chr11 & Satt653, Satt477, Satt592, Satt243 \\
Chr12 & BE806308, Satt197, Satt519, Satt359 \\
Chr13 & Satt635, Satt568, Satt469, Sat_180 \\
Chr14 & Satt663, Sat_417, Satt395 \\
Chr15 & Sat_342, Satt416, Satt318, Sat_424 \\
Chr16 & Satt411, Sat_380, Satt263, Sat_381 \\
Chr17 & Satt285, Satt249, Satt414, Satt244 \\
Chr18 & Sat_333, Satt389, Sat_338, Satt672 \\
Chr19 & Sat_210, Sat_163, Satt138, Satt472 \\
Chr20 & Sat_301, Sat_405, Satt156, Satt229 \\
Chloroplast & Satt419, Satt270, Sat_420 \\
& CSat_005, CSat_024, CSatt028, \\
\hline gamep 3 , CSat_078, SOYCP3) \\
\hline
\end{tabular}

${ }^{a}$ Same as chromosome name (or chloroplast).

${ }^{b}$ Refer to Sayama et al. (2011) and Sasaki et al. (2005).

${ }^{c}$ Xu et al. (2002) used marker.

length, height and width and seed weight were recorded. Measurements were performed to select ten average intact seeds from each plant. The length of the seed was defined as the longest distance across the seed parallel to the hilum, the seed height as the longest distance from the top to bottom of the seed and seed width as the longest distance across the seed perpendicular to the hilum.

\section{DNA extraction and maker analysis}

For plant DNA was extracted from $10 \mathrm{mg}$ seed powder using an automated purification system (BioSprint 96 DNA Plant Kit; Qiagen, Hilden, Germany). Multiplex PCR was performed in a reaction mixture of $5.5 \mu$ l containing $50 \mathrm{nM}$ of each fluorescent primer pair, $5 \mathrm{ng}$ total genomic DNA and $2.5 \mu$ of $2 \times$ Qiagen Multiplex PCR Master Mix (Qiagen) using a GeneAmp PCR System 9700 thermal cycler (Applied Biosystems). The simple sequence repeat (SSR) marker panel for multiplex PCR is shown (Table 1). Subsequently, the amplification protocol and detection of the resulting amplicons using a fluorescence-based DNA sequencer followed the previous report (Sayama et al. 2011).

\section{Statistical analysis}

Allele number per locus was the number of alleles with different sized PCR products amplified by the primer set at each SSR locus. Effective allele $\left(n_{e}\right)$ was described by Kimura and Crow (1964) using the following fomula: $n_{e}=1 /$ $\Sigma p_{i j}{ }^{2}$, where $\mathrm{p}_{i j}$ is the frequency of the $j$ th pattern for the $i$ th marker. The allelic diversity of the SSR polymorphism was calculated according to the diversity index $(H)$ described by Nei (1973) using the following formula: $H=1-\Sigma p_{i}^{2}$, where $p_{i}$ is the same in the case of effective allele calculation.

Phylogenic analysis was performed with the computer program Population 1.2.30 (Langella 1999) using the neighborjoining (NJ) method (Saitou and Nei 1987) based on genetic distance by Nei et al. (1983) with 1,000 bootstraps.

Population structure analysis by a model-based clustering method was performed using the STRUCTURE v2.2.3 program (Prichard et al. 2007). The genotype data were treated as haploid, because soybean is a self-pollinating crop. Clustering analysis was conducted by the admixture and allele frequency correlated model with a linkage model (Falush et al. 2003). In this analysis, the genetic distances between SSR markers were determined with reference to the maps of Hawng et al. (2009). When a certain genotype $(X)$ was supposed, the posterior probabilities $P(K / X)$ were estimated using Markov chain Monte Carlo (MCMC) simulations with a $2 \times 10^{6}$ iteration process following a burn-in period of $2 \times 10^{6}$, where $X$ is a certain genotype in one variety, and $K$ is the number of populations in the tested variety population. $K$ was inferred from the fraction of genetic ancestry and was estimated by ten MCMC simulations. We assigned "founder" landraces to black soybean landraces with membership probabilities $\geq 90 \%$ and "admixed" landraces to landraces with membership probabilities $<90 \%$ for all groups.

\section{Results}

\section{DNA polymorphism in nucleus and chloroplasts}

DNA polymorphism was investigated for 57 landraces of black soybean, including four small seed soybeans originating from the Tanba region compared with 19 representative Japanese landraces.

Several indices for genetic diversity based on nuclear SSR polymorphism among populations originating from different regions are shown (Table 2). Although the numbers of alleles at each marker were not markedly different among populations, the number of effective alleles and the diversity index for the population from Tanba region were smaller than populations from other regions. Furthermore, two groups of black soybean landraces originating from Tanba region were compared to distinguish landraces from the field (field isolates) and landraces conserved at several organizations (conserved accessions). As a result, the number of effective alleles and the diversity index of both groups were not different. Although the varieties of black soybeans with small seeds originating from various regions in Japan were evaluated as one group, the genetic diversity was not large and similar to the population of large seed black soybeans originating from the Tanba region.

The SSR polymorphism in chloroplast DNA was investigated in 76 black soybean landraces for its similarity to nucleic DNA (Table 3). The two genotypes were found in the population of black soybean, and one genotype (B) was 
Table 2. Comparison of genetic diversity in the population of black soybean landraces derived from different regions using nucleic DNA SSR markers

\begin{tabular}{llcccc}
\hline \hline Seed type & Region of origin & No. of races & $\begin{array}{c}\text { No. of alleles/locus } \\
\left(n_{a}\right)\end{array}$ & $\begin{array}{c}\text { No. of effective } \\
\text { alleles/locus }\left(n_{e}\right)\end{array}$ & $\begin{array}{c}\text { Diversity index } \\
(H)\end{array}$ \\
\hline Large & Tanba region (F) & 42 & 4.2 & 1.8 & 0.32 \\
& Tanba region (C) & 11 & 3.0 & 1.7 & 0.33 \\
& Western Japan except Tanba regions & 8 & 3.8 & 2.5 & 0.50 \\
\hline Small & East Japan & 9 & 4.1 & 1.3 & 0.53 \\
\hline Total & - & 6 & 1.5 & 2.0 & 0.18 \\
\hline
\end{tabular}

Tanba region (F): field isolate.

Tanba region $(\mathrm{C})$ : conserved accession.

Black soybean races with small seed bundled together in a group, although these races originated from different regions.

Table 3. Genotype constructed using seven SSR markers of chloroplast DNA in black soybean landraces

\begin{tabular}{|c|c|c|c|c|c|c|c|c|}
\hline \multirow{2}{*}{ Sample } & \multirow{2}{*}{ Genotype } & \multirow{2}{*}{ No. of races } & \multicolumn{6}{|c|}{ SSR markers } \\
\hline & & & CSat_005 & CSat_024 & CSatt028 & Gmcp3 & CSat_078 & $\overline{\text { SOYCP }}$ \\
\hline \multirow[t]{2}{*}{ Landraces } & Type A & 44 & 153 & 276 & 202 & 106 & 144 & 93 \\
\hline & Type B & 32 & 153 & 272 & 202 & 107 & 145 & 94 \\
\hline Ennrei* & Type B & - & 153 & 272 & 202 & 107 & 145 & 94 \\
\hline Harrosoy* & Type B & - & 153 & 272 & 202 & 107 & 145 & 94 \\
\hline
\end{tabular}

* The genotypes of two yellow soybean varieties indicated for reference.

same as those of reference varieties: "Enrei" and "Williams 82". In black soybeans originating from Tanba area, 41 soybeans possessed the chloroplast genome of A type and 16 soybeans possessed B type. On the other hand, among 19 black soybeans originating from areas except Tanba region in Japan, the B type chloroplast genome was found in 15 soybeans and the rate of type B was higher than in populations of black soybeans from Tanba area.

\section{Phylogeny of the population of black soybean}

Based on nuclear SSR polymorphism, phylogenic analysis was performed on the population of black soybean landraces with "Enrei" and "Williams 82" as the outgroup varieties (Fig. 2A). As a result, the population of black soybeans could be classified into six clades. The first and second clades consisted of landraces from Tanba region. In detailed analysis, the first clade could be divided into 11 sub-groups from "a" to " $k$ ". "a" and "b" sub-groups included standard black soybeans with extra large seeds and late filling, which are presently cultivated in the main production areas of Hyogo and Kyoto prefectures. Landraces originating from areas other than Tanba region were mainly classified into clade 3 to 6 , although a few landraces were included in clade 1. The landraces in western Japan were classified into the clade 3 and those in the Kanto-Tozan region and eastern Japan were classified into clade 4. However, bootstrap values were below 70 at ancestral nodes of each clade, and the robustness of each branching was less in this phylogenic analysis.

A comparison of chloroplast genotypes with the position of each landrace on the phylogenic tree by nucleic DNA polymorphism is shown (Fig. 2B). Landraces in clade 1 mainly possessed type A chloroplast genome, although four out of 44 landraces possessed type B chloroplast genome. On the other hand, all landraces in clade 2 possessed type B chloroplast genome. Furthermore, type A chloroplast genome was rarely found in populations of landraces originating from regions other than the Tanba region; therefore, black soybeans with the type A chloroplast genome form a unique population in Japan.

\section{Population structure}

Population structure analysis can clarify the degree of genomic admixture in each landrace by estimating the founder genotype. Therefore, we expected the elucidation of the gene flow into black soybean population as the result of phylogenic analysis. We found that the estimated value of $\mathrm{Pr}$ $(X \mid K)$ was highest for $K=6$ (Table 4); therefore, it was considered that the optimum $K$ value is 6 for clustering in the investigated black soybean population. Each landrace genotype is indicated by a color-coded bar for the clustering groups (Fig. 3A) with the clade number by phylogenic analysis and chloroplast genotypes (Fig. 3B). All landraces derived from Tanba region classified into clades 1 and 2 by phylogenic analysis were distributed into three groups, 1, 2 and 3 by population structure analysis except one landrace. Landraces in clade 1 by phylogenic analysis were mainly divided into group 1 and group 2 by structure analysis. Group 3 in structure analysis included all of the landraces in clade 2 by phylogenic analysis. Especially, landraces in clades of $1 \mathrm{a}, 1 \mathrm{~b}$ and $1 \mathrm{c}$ in the phylogenic tree configure founder population in group 1 population, since these landraces shared 


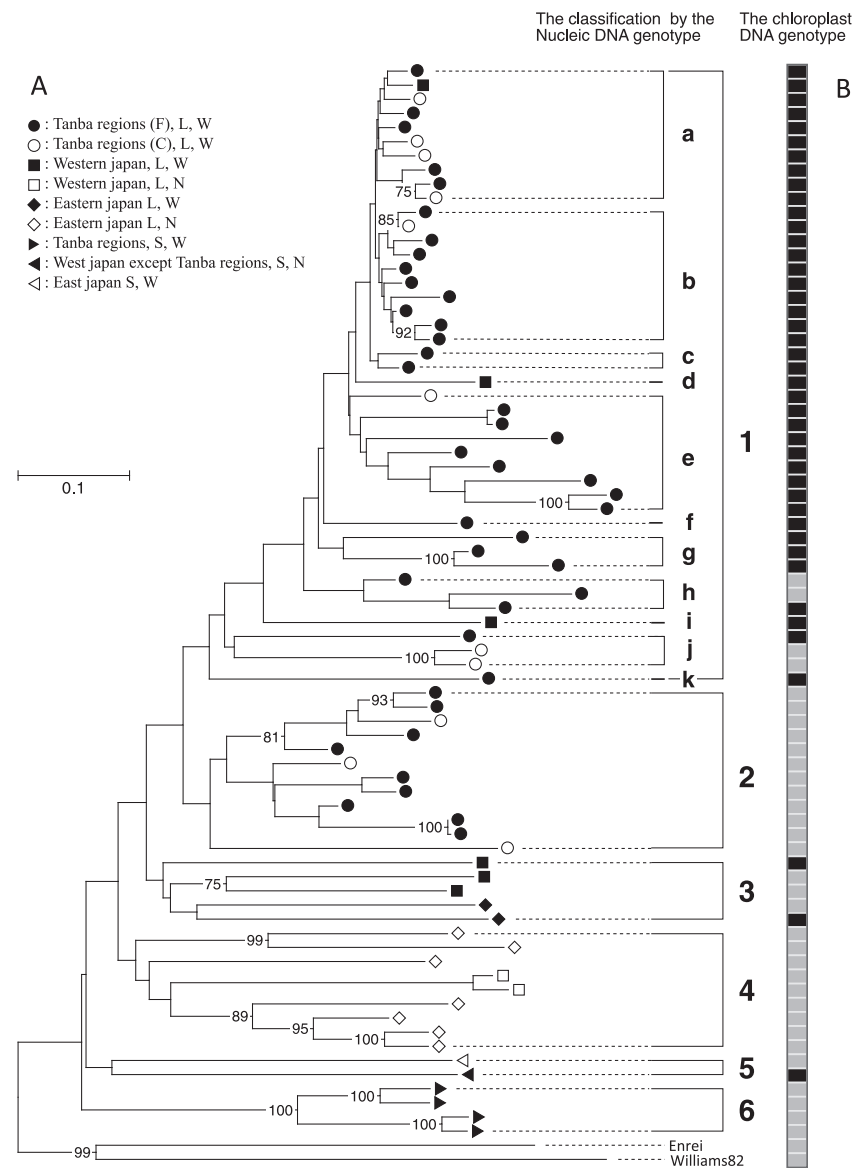

Fig. 2. Classification of black soybean landraces by nucleic DNA genotypes and chloroplast DNA genotypes by SSR polymorphism (A). Landaraces originated from Tanba region are clarified two types: conserved accession (C), field isolate (F). Symbols in the figure indicate the type of landraces by the following features by origin, grain size (L: large seed, S: small seed) and waxy deposit on seed coat (W: with waxy deposit, N: without waxy deposit). Black and grey boxes indicate Type A and B chloroplast DNA genotypes, respectively (B). The numbers in tree diagram indicate the exceeded bootstrap value by 70 .

Table 4. Inferred values of $\mathrm{K}$, the number of populations for black soybean landraces

\begin{tabular}{ccc}
\hline \hline No. of groups $(K)$ & $\operatorname{Ln} \operatorname{Pr}(X \mid K)$ & $\operatorname{Pr}(K \mid X)$ \\
\hline 1 & -5862 & $\sim 0$ \\
2 & -4797 & $\sim 0$ \\
3 & -4261 & $\sim 0$ \\
4 & -4190 & $\sim 0$ \\
5 & -4064 & $\sim 0$ \\
6 & -3996 & 1 \\
7 & -4301 & $\sim 0$ \\
8 & -4613 & $\sim 0$ \\
\hline
\end{tabular}

membership genotypes of group 1 above $96 \%$. On the other hand, several admixed soybeans shared genotypes between landraces in groups 1 and 2 or groups 1 and 3. While dominant genotypes of chloroplast DNA in the populations of groups 1 and 3 were genotype A and B, respectively, the population in group 2 was a combination of landraces with both genotypes of chloroplast DNA. Group 4 was consisted of various landraces: "Chusei-hikariguro" the leading variety in Hokkaido prefecture, "Kurosengoku" and "Wasekurosengoku" characterized by extra small seeds and "Kurohira," characterized by a flattened shape seed with landraces collected in western Japan. These landraces were included in clades 3, 4 and 5 classified by phylogenic analysis. Groups 5 and 6 were composed of landraces included in clades 4 and 5 classified by phylogenic analysis, respectively.

\section{Characteristics of classification group}

The plant growth characteristics of three genotype groups classified by population structure analysis and originating from Tanba region is shown (Table 5). Group 1 and 2 flowered over one week later than group 3. Node and branch numbers in group 1 and 2 were larger than those of group 3; however, the ratios of the branch number to node number were not different among the three groups. On the other hand, pod numbers in group 1 and 2 were fewer than those in group 3. The characteristics of the harvested seed are shown (Table 6). Total seed weight per plant was largest in group 3. The seed weight increased in the order of group 3, 2 and 1. Relating seed weight to, the three seed dimensions also showed differences among the three groups. In particular, seed height and width varied rather than seed length. Comparing the ratios among dimensions, the value in group 1 was closer to 1 than other groups.

The distribution of characteristics described above for each landrace in the three groups is shown as a the scatter diagram in order to verify the characteristics of major landraces cultivated in the Tanba region. In this diagram, the scatter plots were indicated by dividing each group into two sub-groups: founder population $(\mathrm{F})$ and admixture population $(\mathrm{M})$. The landraces in sub-group $1(\mathrm{~F})$ and group 2 were characterized by long flowering periods above 56 days, while the landraces in sub-group 3(F) observed from 49 to 59 days at these periods except one landrace (Fig. 4). In subgroup $1(\mathrm{~F})$, filling period was from 95 to 110 days. On the other hand, many landraces were observed below 100 days in sub-group 3(F), although, two landraces in group 3 were observed with extremely long filling periods more than 120 days. In group 2, a relative wide range of filling period from 90 days to 110 days was observed. Also, in sub-group 1(M), two types of landrace segregated for flowering and filling periods. Landraces of one type showed similar phenotypes for both periods - sub-group 1(F), while those of another type showed equal traits for both periods for major landrace of sub-group $3(\mathrm{~F})$. The distribution of seed weight and the ratio of seed width to length (W/L) is shown (Fig. 5). Seed weight in sub-group $1(\mathrm{~F})$ varied from $0.68 \mathrm{~g}$ to $0.92 \mathrm{~g}$, and seed weights were partitioned at a threshold of $0.6 \mathrm{~g}$ between sub-group 1(F) and group 3. The distribution of seed width to seed length ratio in sub-group $1(\mathrm{~F})$ was higher, and the range was relatively smaller than in sub-group $3(\mathrm{~F})$. 

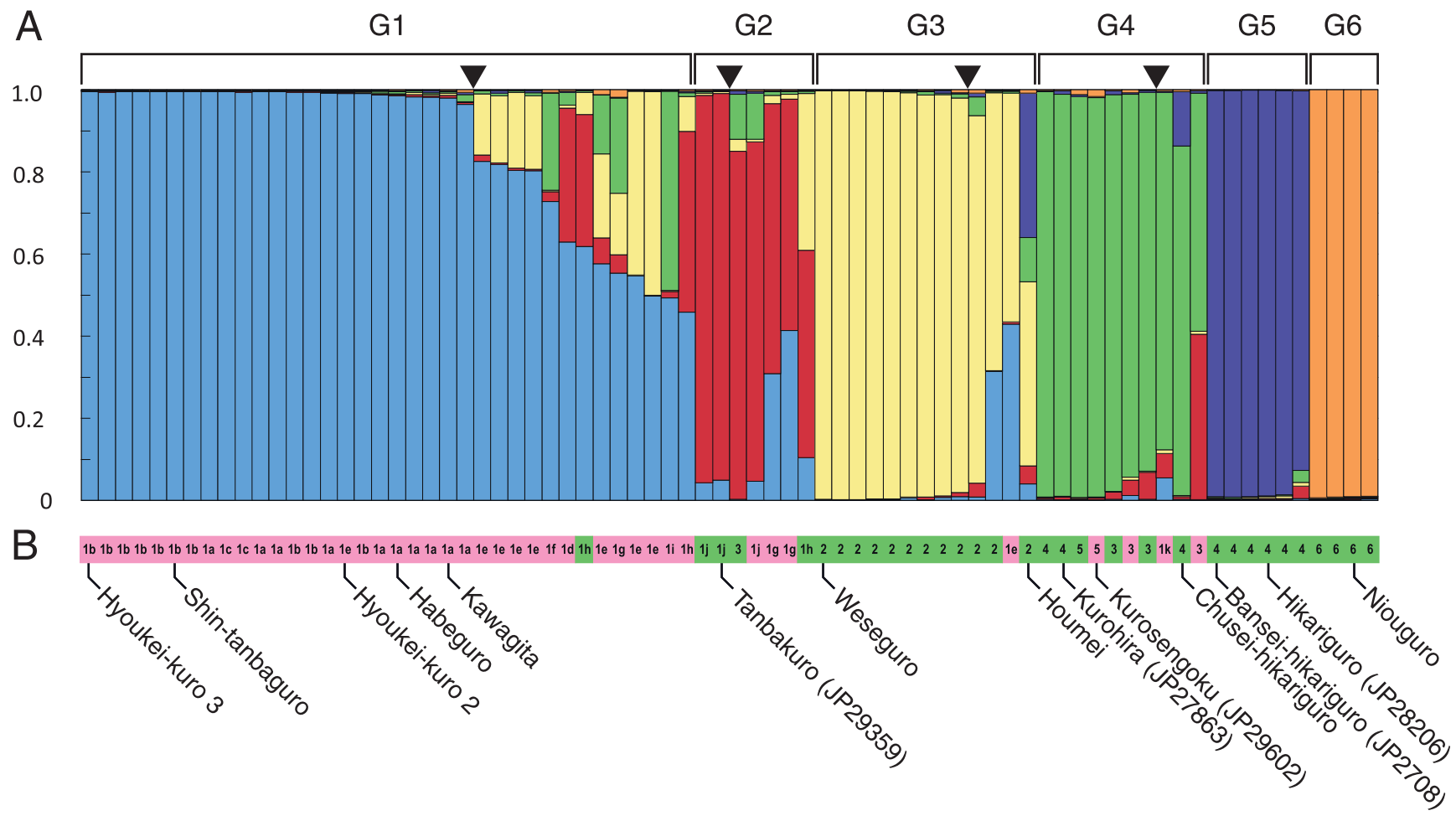

Fig. 3. A shows the population structure involved in diverse black soybean landraces. Color bar indicates the genotypes inferred from the clustering groups. B shows the clade number at Fig. 1 by phylogenic analysis, and chloroplast DNA genotype of each landraces. Pink and green backgrounds indicate Type A and B chloroplast DNA genotypes, respectively. Triangles indicate the limits of membership probabilities $\geq 90 \%$ in each group.

Table 5. Characteristics of plant growth in each genotype group of black soybean landraces

\begin{tabular}{|c|c|c|c|c|c|c|c|c|c|c|}
\hline $\begin{array}{l}\text { Genotype } \\
\text { Group }\end{array}$ & $\begin{array}{l}\text { No. of } \\
\text { races }\end{array}$ & $\begin{array}{l}\text { Flowering } \\
\text { period (day) }\end{array}$ & $\begin{array}{l}\text { Maturation } \\
\text { period (day) }\end{array}$ & $\begin{array}{l}\text { Stem length } \\
(\mathrm{cm})\end{array}$ & $\begin{array}{l}\text { Stem diameter } \\
(\mathrm{mm})\end{array}$ & $\begin{array}{l}\text { Node number } \\
\text { (N) }\end{array}$ & $\begin{array}{c}\text { Branch } \\
\text { number (B) }\end{array}$ & $\mathrm{B} / \mathrm{N}$ & $\begin{array}{l}\text { Pod number } \\
\text { (P) }\end{array}$ & $\mathrm{P} / \mathrm{N}$ \\
\hline 1 & 30 & $59 \mathrm{a}$ & $105 \mathrm{a}$ & $80.0 \mathrm{a}$ & $16.7 \mathrm{a}$ & $17.9 \mathrm{a}$ & $11.4 \mathrm{a}$ & $0.64 \mathrm{a}$ & $188 \mathrm{a}$ & $10.3 \mathrm{a}$ \\
\hline 2 & 5 & $59 \mathrm{a}$ & $104 \mathrm{a}$ & $81.4 \mathrm{a}$ & $17.3 \mathrm{~b}$ & $17.9 \mathrm{a}$ & $11.2 \mathrm{ab}$ & $0.63 \mathrm{a}$ & $193 \mathrm{ab}$ & $10.8 \mathrm{a}$ \\
\hline 3 & 13 & $51 \mathrm{~b}$ & $100 \mathrm{a}$ & $70.4 \mathrm{a}$ & $15.8 \mathrm{a}$ & $16.2 \mathrm{~b}$ & $10.0 \mathrm{~b}$ & $0.61 \mathrm{a}$ & $234 \mathrm{~b}$ & $14.4 \mathrm{~b}$ \\
\hline
\end{tabular}

Genotype groups are shown as classified by population structure analysis.

Data are presented as the mean of lines in each genotype group.

Means followed by the same letters were not significantly different from each other at the $5 \%$ level within a column on each line (Tukey-Kramer multiple comparison test).

Table 6. Characteristics of harvested seed in each genotype group of black soybean landrace

\begin{tabular}{|c|c|c|c|c|c|c|c|c|c|}
\hline $\begin{array}{c}\text { Genotype } \\
\text { Group }\end{array}$ & $\begin{array}{l}\text { No. of } \\
\text { races }\end{array}$ & $\begin{array}{c}\text { Total seed } \\
\text { weight }(\mathrm{g} / \text { plant })\end{array}$ & $\begin{array}{c}\text { Seed weight } \\
\text { (g) }\end{array}$ & $\begin{array}{l}\text { Seed length } \\
(\mathrm{mm})(\mathrm{L})\end{array}$ & $\begin{array}{l}\text { Seed height } \\
(\mathrm{mm})(\mathrm{H})\end{array}$ & $\begin{array}{c}\text { Seed width } \\
(\mathrm{mm})(\mathrm{W})\end{array}$ & $\mathrm{H} / \mathrm{L}$ & $\mathrm{W} / \mathrm{L}$ & $\mathrm{W} / \mathrm{H}$ \\
\hline 1 & 30 & $150 \mathrm{a}$ & $0.73 \mathrm{a}$ & $11.2 \mathrm{a}$ & $10.6 \mathrm{a}$ & $9.4 \mathrm{a}$ & $0.94 \mathrm{a}$ & $0.84 \mathrm{a}$ & $0.89 \mathrm{a}$ \\
\hline 2 & 5 & $159 \mathrm{a}$ & $0.63 \mathrm{~b}$ & $11.2 \mathrm{a}$ & $10.1 \mathrm{~b}$ & $8.7 \mathrm{~b}$ & $0.90 \mathrm{~b}$ & $0.78 \mathrm{~b}$ & $0.87 \mathrm{ab}$ \\
\hline 3 & 13 & $173 \mathrm{~b}$ & $0.51 \mathrm{c}$ & $10.4 \mathrm{~b}$ & $9.5 \mathrm{c}$ & $8.0 \mathrm{c}$ & $0.92 \mathrm{~b}$ & $0.78 \mathrm{~b}$ & $0.85 \mathrm{~b}$ \\
\hline
\end{tabular}

Data are the same as in Table 5.

\section{Discussion}

Japanese soybean varieties are usually classified into two biotypes summer and autumn soybeans. Nagata (1959, 1960) suggested that both types of cultivar have adapted to local cultivation patterns in Japan, and have become established through different phyletic lines. Black soybean culti- vars are also differentiated by growth type depending on the cultivation area in Japan. The black soybean landrace population originating from Tanba region was identified as "Tanbaguro" or "Tanbakuro", and classified as an autumn soybean type that is characterized by late flowering and extremely long filling periods. On the other hand, as one of the major varieties of black soybean in Hokkaido, "Chusei- 


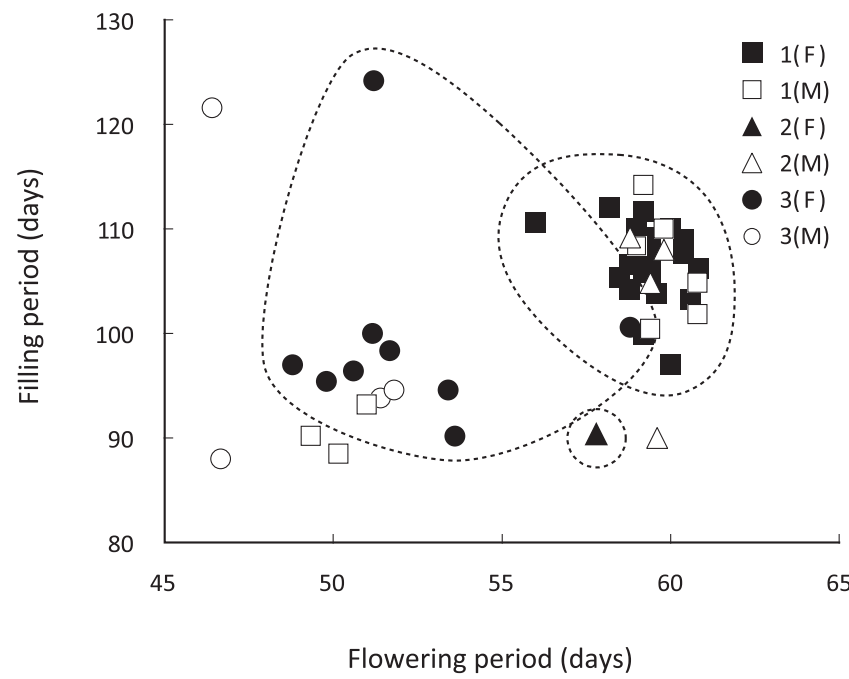

Fig. 4. Scatter plot of flowering periods and filling periods of black soybean landraces. Enclosed circle with a broken line indicate the distribution area of founder landraces in each group. The landraces were classified into two sub-groups: fouder (F) and admixture (M) in group $1-3$.

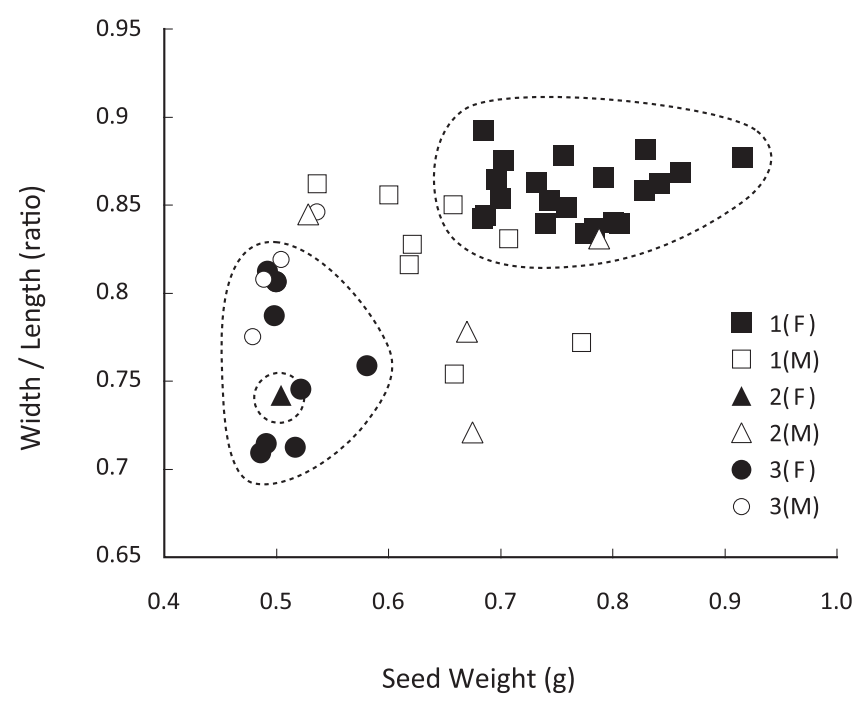

Fig. 5. Scatter plot of seed weight and ratio of seed width to length of black soybean landraces. Enclosed circle with broken line indicates the same area as in Fig. 4. Explanatory notes are also indicated the same classifications in Fig. 4.

Hikariguro" is characterized by extremely early maturing. However, it is well known that the characters for insensitivity to long daylength and maturity are controlled by some genetic factors (Abe et al. 2003, Buzzell 1971, Buzzell and Voldeng 1980, Cober and Voldeng 2001, Saindon et al. 1989).

Apart from the phenotypic classification, we evaluated the genetic diversity of black soybean landraces in Japan by the length polymorphism of SSR markers in genomic DNA in this study. The comparison of genetic diversity, we used the two kinds samples for black soybean derived from Tanba
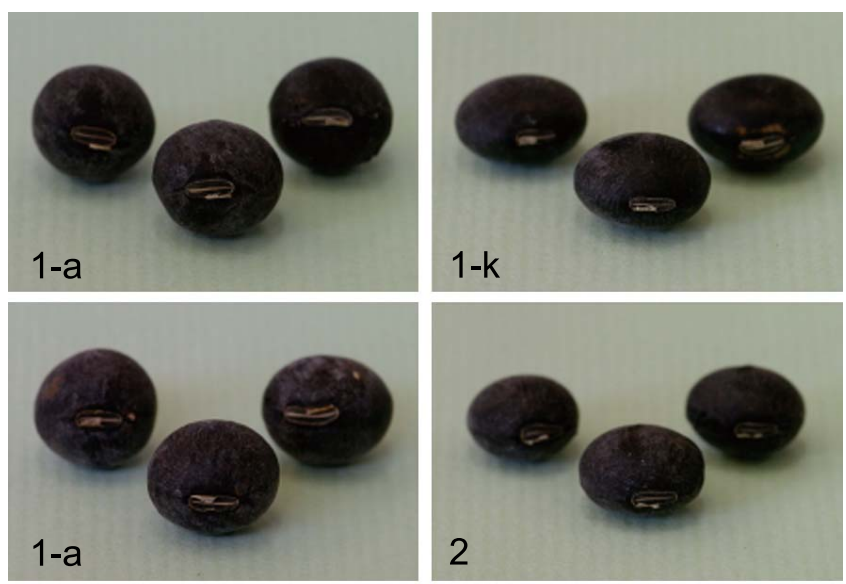

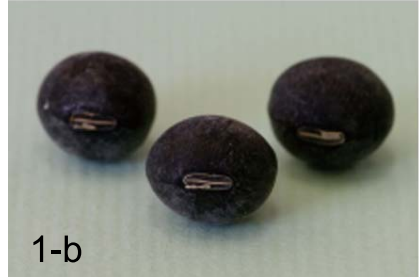

Group 1

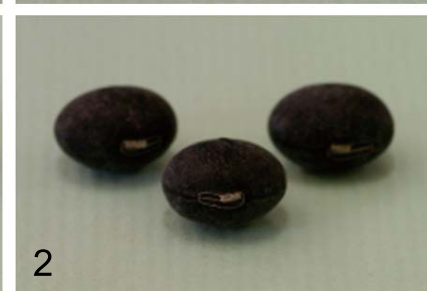

Group 3
Fig. 6. Seed shapes of black soybean landraces classified by population structure analysis. The texts in figure indicated the clade on the phylogenic analysis.

region: field isolates and conserved accessions at several organizations. However, several indices of genetic diversity were not distinguished between these samples, indicating that the genetic diversity of black soybeans originating from Tanba region is still conserved in this region. It was considered from this result that black soybean landraces in this region have been isolated for many years. Accordingly, we analyzed the phyletic relationship and differentiation of the black soybean landraces in Japan. At the result, we found that the black soybean population originating from Tanba region could be distinguished from those of other regions of Japan, and was derived from two or more different ancestors, since two chloroplast genotypes were found in the population of black soybeans from Tanba region. Additionally, the geographic differentiation of soybean landraces could be explained by phylogenic analysis using nucleic DNA polymorphism.

Interestingly, genotype A chloroplast was only identified in one group of Tanba region black soybeans with a few exceptions, while genotype B was widely distributed among other Japanese black soybean populations with another group of Tanba region black soybeans in the present study. $\mathrm{Xu}$ et al. (2002) surveyed the variation in the chloroplast genome using six SSR markers, and three genotypes were found in Japanese soybean cultivars. In this study, we used two common markers of the six in their report. As a result, it was considered that type B is the dominant type [no. 49 genotype of Xu et al. (2002)], and, type A chloroplast in our study is no. 20 genotype of Xu et al. (2002). Xu et al. (2002) 
reported that the soybean varieties of this genotype are distributed in southern Japan, and are detected in wild soybean rather than cultivated soybean. Therefore, it is considered that the origin of "Tanbaguro" with genotype A is different from other cultivated soybeans in Japan.

In this study, we grouped black soybean landraces by two methods: distance-based phylogenic analysis and modelbased population structure analysis, for nuclear DNA polymorphism. Prichard et al. (2000) indicated that the former method is suitable to understand the genetic relationships among accessions, however, it is difficult to assess how confident we should be that the clusters obtained in this way are meaningful. Practically, we found the population structure in the landraces originating form Tanba region with different founders and admixture by structure analysis, although, corresponding results were obtained for clustering.

Black soybean landraces in Tanba region was classified into three groups by population structure analysis. It is considered that groups 1 and 3 are derived from different ancestors, because each group had different chloroplast genomes. In group 2, two founder landraces also possess Type B chloroplast genome, although, both types of chloroplast genotype were observed as admixture in other landraces. Based on the nuclear genotype, admixture landraces were found between group 1 and 2 or group 1 and 3 , although there are few genotypes shared between group 2 and 3 . This fact suggests that the landraces in group 1 are widely cultivated in Tanba region, while the landraces in group 2 and 3 were cultivated at localized area, supposing that the admixture landraces were derived form hybridization. Comparing phenotypes among genotype groups, several characters were clearly distinguished between founder landraces in group 1 and 3. Especially, the founder landraces in group 1 have larger and more spherical seeds than those in group 3 (Fig. 6). Landraces in admixture landraces in group 1 and 2 segregated for characters of flowering, seed filling and seed shape and weight, while founder landraces in group 1 were stabled for each character. These observations also suggested that gene flow events have occurred between different founder landraces. QTLs were recently found for the flowering and seed filling periods and seed shape using DNA markers by several research groups (Cheng et al. 2011, Liu and Abe 2010, Salas et al. 2006, Watanabe et al. 2009). Alleles of these QTLs between groups of black soybean landraces in Tanba region should be clarified.

In this study, we found that black soybeans in Tanba and neighboring regions have wide genetic diversity. Soybeans are closely intertwined with traditional food culture in Japan, but commercial quality demands have changed over time. On the other hand, the genetic diversity of landrace populations is declining for agricultural products, extending agricultural product marketing; therefore, continued collecting of local genetic resources and clarification of their genetic background is imperative.

\section{Acknowledgements}

The authors are grateful to Dr. T. Nakasaki, Kyoto University, Odagaki Inc., Kyoto Prefectural Agriculture, Forestry and Fisheries Technology Center, Hokkaido Central Agricultural Experiment Station, and the Genebank of the National Institute of Agrobiological Sciences for providing the germplasm. This work was partially supported by [Genomics for Agricultural Innovation (DD-3260)] of the Ministry of Agriculture, Forestry and Fisheries of Japan.

\section{Literature Cited}

Abe,J., D.Xu, A.Miyano, K.Komatsu, A.Kanazawa and Y.Shimamoto (2003) Photoperiod-insensitive Japanese soybean landraces differ at two maturity loci. Crop Sci. 43: 1300-1304.

Buzzell, R.I. (1971) Inheritance of a soybean flowering response to fluorescent-daylength conditions. Can. J. Genet. Cytol. 13: 703707.

Buzzell, R.I. and H.D. Voldeng (1980) Inheritance of insensitivity to long daylength. Soybean Genet. Newsl. 7: 26-29.

Cheng,L., Y.Wang, C.Zhang, C.Wu, J.Xu, H.Zhu, J.Leng, Y.Bai, R.Guan, W.Hou et al. (2011) Genetic analysis and QTL detection of reproductive period and post-flowering photoperiod responses in soybean. Theor. Appl. Genet. 123: 421-429.

Cober,E.R. and H.D.Voldeng (2001) A new soybean maturity and photoperiod-sensitivity locus linked to E1 and T. Crop Sci. 41: 698-701.

Falush,D., M.Stephens and J.K.Pritchard (2003) Inference of population structure using multilocus genotype data: linkage loci and correlated allele frequencies. Genetics 164: 1567-1587.

George,N.U., W.J.Kenworthy, J.M.Costa, P.B.Cregan and J.Alvernaz (2003) Genetic diversity of soybean cultivars from China, Japan, North America, and North American ancestral lines determined by amplified fragment length polymorphism. Crop Sci. 43: 18581867.

Hirata,T., J.Abe and Y.Shimamoto (1999) Genetic structure of the Japanese soybean population. Gen. Res. Crop Evol. 46: 441-453.

Hatanaka, T., S.Yoshida, M.Yagawa, A.Shibata, H.Kosaka and T. Toda (2008) The estimation of relatedness for the standardization of Tanbaguro type black soybean varieties by SSR marker. J. Crop Res. 50: 13-24.

Hwang, T.Y., Y.Nakamoto, I.Kono, H.Enoki, H.Funatsuki, K.Kitamura and M.Ishimoto (2008) Genetic diversity of cultivated and wild soybeans including Japanese elite cultivars as revealed by length polymorphism of SSR markers. Breed. Sci. 58: 315-323.

Hwang, T.Y., T.Sayama, M. Takahashi, Y.Takada, Y. Nakamoto, H.Funatsuki, H.Hisano, S.Sasamoto, S.Sato, S. Tabata et al. (2009) High-density integrated linkage map based on SSR markers in soybean. DNA Res. 16: 213-225.

Kimura,M. and J.F.Crow (1964) The number of alleles that can be maintained in a finite population. Genetics 49: 725-738.

Kosaka,H., T.Hatanaka, S. Yoshida and T.Toda (2009) Identification of black soybean (Glycine max) Tanbaguro and processed foods by simple sequence repeat analysis. Nippon Shokuhin Kagaku Kaishi 56: 119-128.

Langella,O. (1999) Populations, 1.2.30. French National Center for Scientific Research (CNRS) http://bioinformatics.org/ tryphon/ populations/

Liu,B. and J.Abe (2010) QTL mapping for photoperiod insensitivity 
of a Japanese soybean landrace Sakamotowase. J. Heredity 101: 251-256.

Nagata,T. (1953) Some considerations on the culture of a black soybean, Tamba-Kuro-Daizu. Sci. Reports of Hyogo Univ. Agr. 1: 9 12.

Nagata,T. (1959) Studies on the differentiation of soybeans in the world, with special regard to that in southeast Asia: 2. Origin of culture and paths of dissemination of soybeans, as considered by distributions of their summer vs. autumn soybean habit and plant habit. Proc. Crop. Sci. Soc. of Jpn. 28: 79-84.

Nagata,T. (1960) Studies on the differentiation of soybeans in Japan and the world. Mem. Hyogo Univ. Agric., Agron. Ser. 4: 63-102.

Nei,M. (1973) Analysis of gene diversity in subdivided populations. Proc. Nat. Acad. Sci. 70: 3321-3323.

Nei,M., F.Tajima and Y.Tateno (1983) Accuracy of estimated phylogenetic trees from molecular data II. Gene frequency data. J. Mol. Evol. 19: 153-170.

Pritchard,J.K., M.Stephens and P.Donnelly (2000) Inference of population structure using multilocus genotype data. Genetics 155: 945959.

Pritchard,J.K., W.Wen and D.Falush (2007) STRUCTURE ver. 2.2 University of Chicago, USA. http//:pritch.bsd.uchicago.edu/

Saindon, G., H.D.Voldeng, W.D. Beversdorf and R.I.Buzzell (1989) Genetic control of long daylength response in soybean. Crop Sci. 29: 1436-1439.

Saitou,N. and M.Nei (1987) The neighbor-joining method: a new method for reconstructing phylogenetic trees. Mol. Biol. Evol. 4: 406-425.

Salas,P., J.C.Oyarzo-Llaipen, D. Wang, K.Chase and L.Mansur (2006) Genetic mapping of seed shape in three populations of recombinant inbred lines of soybean (Glycine max L. Merr.) Theor. Appl. Genet. 113: 1459-1466.

Sasaki,C., S.B.Lee, H.Daniell, T.C.Wood, J.Tomkins, H.G.Kim and R.K.Jansen (2005) Complete chloroplast genome sequence of Glycine max and comparative analyses with other legume genomes. Plant Mol. Boil. 59: 309-322.

Sayama,T., T.Y.Hwang, K.Komatsu, Y.Takada, M.Takahashi, S.Kato, H.Sasama, A.Higashi, Y.Nakamoto, H.Funatsuki et al. (2011) Development and application of a whole-genome simple sequence repeat panel for high-throughput genotyping in soybean. DNA Res. 18: 107-115.

Wang,L., R.Guan, L.Zhangxiong, R.Chang and L.Qiu (2006) Genetic diversity of Chinese cultivated soybean revealed by SSR markers. Crop Sci. 46: 1032-1038.

Watanabe,S., R.Hideshima, Z.Xia, Y.Tsubokura, S.Sato, Y.Nakamoto, N. Yamanaka, R.Takahashi, M.Ishimoto, T.Anai et al. (2009) Map-based cloning of the gene associated with the soybean maturity locus E3. Genetics 182: 1251-1262.

Xu,D.H., J.Abe, J.Y.Gai and Y.Shimamoto (2002) Diversity of chloroplast DNA SSRs in wild and cultivated soybeans: evidence for multiple origins of cultivated soybean. Theor. Appl. Genet. 105: 645-653. 